\title{
SARS-COV-2 infection in pregnant women and newborns in a Spanish cohort (GESNEO-COVID) during the first wave
}

Itzíar Carrasco $0^{1,2^{*}}$, Mar Muñoz-Chapuli³, Sara Vigil-Vázquez ${ }^{4}$, David Aguilera-Alonso ${ }^{1,2,5}$, Concepción Hernández ${ }^{3}$, César Sánchez-Sánchez ${ }^{6}$, Cristina Oliver ${ }^{3}$, Mónica Riaza ${ }^{7}$, Marta Pareja ${ }^{8}$, Olga Sanz ${ }^{9}$, Beatriz Pérez-Seoane ${ }^{10}$, Juan López ${ }^{11}$, Elena Márquez ${ }^{12}$, Sara Domínguez-Rodríguez ${ }^{13}$, Alicia Hernanz-Lobo 1,2,5, Juan Antonio De León-Luis, ${ }^{2,3}$, Manuel Sánchez-Luna ${ }^{2,4}$ and María Luisa Navarro 1,2,5

\begin{abstract}
Background: Knowledge about SARS-CoV-2 infection in pregnancy and newborns is scarce. The objective of this study is to analyse clinical and epidemiological characteristics of a cohort of women infected with SARS-CoV-2 during pregnancy and their newborns exposed to SARS-CoV-2 during gestation.

Methods: Multicentric observational study of Spanish hospitals from the GESNEO-COVD cohort, participants in RECLIP (Spanish Network of Paediatric Clinical Assays). Women with confirmed SARS-CoV-2 infection by PCR and/or serology during pregnancy, diagnosed and delivering during the period 15/03/2020-31/07/2020 were included. Epidemiological, clinical, and analytical data was collected.

Results: A total of 105 pregnant women with a median of 34.1 years old (IQR: 28.8-37.1) and 107 newborns were included. Globally, almost 65\% of pregnant women had some COVID-19 symptoms and more than 43\% were treated for SARS-COV-2. Overall, 30.8\% of pregnant women had pneumonia and 5 (4.8\%) women were admitted to the intensive care unit needing invasive mechanical ventilation. There was a rate of $36.2 \%$ of caesarean sections, which was associated with pneumonia during pregnancy (OR: 4.203, Cl 95\%: 1.473-11.995) and lower gestational age at delivery (OR: 0.724, Cl 95\%: 0.578-0.906). The prevalence of preterm birth was 20.6\% and prematurity was associated with pneumonia during gestation (OR: 6.970, Cl95\%: 2.340-22.750) and having a positive SARS-CoV-2 PCR at delivery (OR: 6.520, C195\%: 1.840-31.790). All nasopharyngeal PCR in newborns were negative at birth and one positivized at 15 days of life. Two newborns died, one due to causes related to prematurity and another of unexpected sudden death during early skin-to-skin contact after delivery.
\end{abstract}

Conclusions: Although vertical transmission has not been reported in this cohort, the prognosis of newborns could be worsened by SARS-CoV-2 infection during pregnancy as COVID-19 pneumonia increased the risk of caesarean section deliveries and preterm births.

Keywords: COVID-19, Caesarean section, Morbidity, Mortality, Newborn, Pneumonia, Pregnancy, Preterm birth, SARS-COV-2

\footnotetext{
* Correspondence: itziiaarcg@gmail.com

${ }^{1}$ Paediatric Infectious Disease Unit, Instituto de Investigación Sanitaria Gregorio Marañón, Madrid, Spain

${ }^{2}$ Facultad de Medicina Universidad Complutense de Madrid, Madrid, Spain

Full list of author information is available at the end of the article
}

C C The Author(s). 2021 Open Access This article is licensed under a Creative Commons Attribution 4.0 International License, which permits use, sharing, adaptation, distribution and reproduction in any medium or format, as long as you give appropriate credit to the original author(s) and the source, provide a link to the Creative Commons licence, and indicate if changes were made. The images or other third party material in this article are included in the article's Creative Commons licence, unless indicated otherwise in a credit line to the material. If material is not included in the article's Creative Commons licence and your intended use is not permitted by statutory regulation or exceeds the permitted use, you will need to obtain permission directly from the copyright holder. To view a copy of this licence, visit http://creativecommons.org/licenses/by/4.0/. The Creative Commons Public Domain Dedication waiver (http://creativecommons.org/publicdomain/zero/1.0/) applies to the data made available in this article, unless otherwise stated in a credit line to the data. 


\section{Introduction}

Severe acute respiratory syndrome coronavirus 2 (SARS$\mathrm{CoV}-2$ ) is a novel beta coronavirus that causes coronavirus disease 2019 (COVID-19), a severe infectious respiratory disease. SARS-CoV-2 first case was reported on December 31, 2019, in Wuhan (China) and has since spread worldwide. The World Health Organization declared an International Emergency on January 30 [1], and a global pandemic on March 11th, 2020 [2]. First SARS-CoV-2 confirmed case in Spain was declared on January 31st, 2020. Up to October 22nd, 2020, 40,652,097 cases of SARS-CoV2 infection have been confirmed and 1,122,036 SARS-CoV2 related deaths have been reported worldwide (Word Health Organization).

The anatomical, physiological and immunological changes accompanying pregnancy may raise pregnant women's susceptibility to viral pathogens and increase the risk of developing severe pneumonia [3, 4] . Evidence from other coronavirus infections, such as SARS-CoV or MERS-CoV, suggests that infected pregnant women might be more susceptible to adverse outcomes, including intubation, intensive care unit (ICU) admission, renal failure and death [5]. Hantoushzadeh et al. suggested that pregnant women SARS-CoV-2 infected during the second or third trimester of their pregnancy may experience clinical complications and die [6].

There is insufficient data available to evaluate the effects of SARS-CoV-2 infection on pregnant women and pregnancy outcomes and the prevalence of perinatal complications is still unknown. It has been reported that the most common symptoms of SARS-CoV-2 infection in women during pregnancy are fever, cough, dyspnoea, fatigue and myalgia [7], similar to most frequent symptoms in other adults [8]. The severity of the infection in adults is associated with certain risk factors such as hypertension, obesity, chronic lung disease, diabetes mellitus and cardiovascular diseases [9]. Sentilhes et al. [10] have found that maternal age, obesity, hypertension or diabetes may increase the risk of pregnant women with COVID-19 morbidity, but these factors are not described in detail in the published series of pregnant women. The rate of severe pneumonia in pregnant women has been reported in a wide range from 0 to $14 \%$ [11].

The possibility of vertical and perinatal transmission is still controversial [12]. There are some studies testing newborns from women infected during pregnancy at birth by RT-PCR (Real-Time Polymerase Chain Reaction) [13-16], other publications have reported several cases of SARS-CoV-2 infected newborns [17-22] and potential mechanisms of perinatal transmission have been reviewed [12]. SARS-CoV-2 has been found in the placenta by RT-PCR [23, 24] and by electron microscopy [25] but newborns in those studies were not infected. The vaginal fluid has also been tested by RT-PCR, without SARS-CoV-2 detection [26]. Salvatore et al. [27] support that if correct hygiene precautions are taken perinatal infection is unlikely to occur. Intrauterine and peripartum transmission risks from mother to child are unknown and there is no clear evidence on mode and timing of delivery [28]. Little is known about the consequences of SARS-CoV-2 infection in newborns. A case of late-onset infection in a newborn has been reported, suggesting the need to follow up newborns from mothers infected during delivery [19]. It has been suggested that the strong innate immunity of children leads to better control of infection at the entry site [29].

The main objective of the study is to describe clinical and analytical characteristics of SARS-CoV-2 infection in a cohort of infected pregnant women during the first wave of the pandemic. Secondary objectives are the analysis of delivery outcomes and perinatal and postnatal transmission of SARS-CoV-2 infection.

\section{Methods}

A prospective, multicentric, observational study from Spanish GESNEO-COVID cohort. Inclusion criteria were: i) women with confirmed SARS-CoV-2 infection by a positive RT-PCR result during pregnancy on a respiratory specimen (nasopharyngeal swab), by the detection of IgG or/and IgM antibodies in serum or by high clinical suspicions even if RT-PCR or/and antibodies in serum were negative; ii) delivery during 15/03/2020-31/07/2020; iii) inclusion of their newborns. Exclusion criteria were women with miscarriage, foetal death and dead newborns.

The first wave of the pandemic in our cohort comprehended from March 15th 2020 to July 31st 2020. During the first month of the study, due to the diagnostic indications and the scarcity of available tests at the hospitals, only symptomatic women were screened for the infection. As the pandemic was going forward, in many hospitals pregnant women admitted for delivery were tested for SARS-CoV-2 infection through a screening program both by active infection (RT-PCR in nasopharyngeal swab) and past infection (IgG antibodies in serum). Thus, asymptomatic women testing positive for active or past SARS-CoV-2 infection at delivery after April 15th were also included in the study.

Preterm birth was defined as newborn birth at less than 37 weeks of gestation. Newborns were considered small for their gestational age (SGA) when their weigh was equal or less than 10th percentile according to Intergrowth and Fenton criteria. If the maternal clinical condition was acceptable/good, newborns were not separated from their mothers after birth and maternal breastfeeding was recommended [30]. Newborns were tested for SARS-CoV-2 by RT-PCR on nasopharyngeal swabs at $24 \mathrm{~h}$ and 15 days of life. Chest X-ray was performed when indicated by the clinical situation and after 
informed consent. Data was retrieved from each hospital's electronic medical history and was collected at birth using REDCap (Research Electronic Data Capture) system [31], hosted on a secure server at the Gregorio Marañón Research Institute in Madrid. Socio-demographic, clinical, analytical and virological data from infected women at diagnosis were collected. Obstetric data and delivery outcomes from infected women and characteristics and clinical conditions from newborns were collected.

The study was approved by the Ethics Committee from Hospital Gregorio Marañón and patients informed consent were obtained before inclusion.

\section{Statistical analyses}

Continuous variables were presented with median and interquartile ranges (IQR). Categorical variables were presented as total counts and percentages (\%). Chisquare test or Fisher test, as appropriate, were performed to compare categorical variables and U Mann-Whitney test to compare continuous variables. We assessed the association of clinical, laboratory and demographic variables with gestational pneumonia, caesarean and prematurity using multivariable analysis. Variable selection for the models was done by a backwards stepwise procedure using Akaike index criterion. A $p$-value less than 0.05 was considered statistically significant. All analyses were conducted in IBM-SPSS Statistics Version 25.0 (Armonk, NY: IBM Corp) and R Software.

\section{Results}

A total of 105 infected pregnant women and 107 newborns (two twin births) were included from GESNEOCOVID cohort.

\section{Description of SARS-CoV-2 infected women during pregnancy}

The median age of pregnant women at delivery was 34.1 (IQR: 28.8-37.1) years. More detailed characteristics are shown in Table 1. More than half (59.6\%) were Caucasian and $28.8 \%$ of them were from Latin America. No woman was diagnosed during the first trimester, $6.7 \%$ of the women were diagnosed during the second trimester of pregnancy and $93.3 \%$ during the third trimester. There were $51.9 \%$ SARS-CoV-2 RT-PCR positive tests at delivery. Overall, $34.3 \%$ of women had some comorbidity: $6.7 \%$ were obese, $1.9 \%$ presented hypertension, $1.9 \%$ asthma, $6.7 \%$ gestational diabetes, $10.5 \%$ gestational hypothyroidism, $1.9 \%$ immunosuppression and $10.5 \%$ presented other comorbidities. No pre-eclampsia was reported.

\section{Effects of SARS-CoV-2 infection during pregnancy in pregnant women and delivery outcomes}

Two thirds (64.8\%) of SARS-CoV-2 infected pregnant women had COVID-19 symptoms. The most common symptoms were fever (36.2\%), cough (35.2\%), and dyspnoea (19.0\%). Less frequent symptoms included myalgia (13.3\%), anosmia (12.4\%), headache (9.5\%), rhinorrhoea (8.6\%), vomiting (6.7\%), diarrhoea (5.7\%) and other symptoms (10.5\%).

Chest X-ray was performed in 52 women and 32 (61.5\%) of them had signs compatible with pneumonia. Overall, radiological images suggested bilateral pneumonia in 20 women and unilateral pneumonia in 12. All 32 pneumonia cases were diagnosed during the first month of the study (15 May-15 April 2020) (Fig. 1). Five women with severe pneumonia and positive RT-PCR at delivery required admission to the Intensive Care Unit for a median of 10.0 (IQR: 6.5-18.5) days, requiring invasive mechanical ventilation. There was no maternal mortality reported.

In the laboratory analyses increased concentrations of $\mathrm{D}$ dimer, higher values of liver enzyme alanine aminotransferase and lower count of lymphocytes were found in women with pneumonia compared with women who did not develop pneumonia (Table 2). Obesity was also associated with a higher risk of pneumonia. Lymphopenia $(<1500$ lymphocytes $/ \mathrm{ml}$ ) and a lower gestational age at diagnosis were potential predictors for pneumonia in the multivariable analysis (Fig. 2).

Overall, 46 women $(43.8 \%)$ received treatment under study for COVID-19. Different treatment options were used and sometimes combined; antiviral treatment: lopinavir/ritonavir (28 women), gamma interferon (4 women) and remdesivir ( 2 women); anti-malarial: hydroxychloroquine (33 women); antibiotic treatment: ceftriaxone ( 25 women) and other antibiotics (16 women); immunomodulatory drugs: systemic corticoids (8 women) and tocilizumab (4 women); and other treatments (13 women). $61.5 \%$ of women received treatment before delivery and $38.5 \%$ received treatment the day of delivery or after, including both women receiving remdesivir. Treatments were safe for both mother and newborns.

Globally, 38 (36.2\%) of pregnant women had a caesarean delivery and the indication for caesarean section was severe COVID-19 for $28.9 \%$ of them. Pregnant women with pneumonia had a 5 -fold increased risk of caesarean sections and premature newborns than those without pneumonia in the univariable analysis (OR:5.0 [2.0;12.2], $p$-value $<0.001$ ) (Table 3). Pneumonia and lower gestational age at delivery were associated with caesarean section as predictor factors in the multivariable analysis (Fig. 2). The women with pneumonia were 4 times at greater risk than those without pneumonia (OR: 4.2[1.47-11.99], $p=0.007$ ) adjusted for gestational age at diagnosis and delivery. All women admitted to the ICU had caesarean sections due to the severity of the disease. 
Table 1 Characteristics of women infected with SARS-CoV-2 during pregnancy by pneumonia

\begin{tabular}{|c|c|c|c|c|c|}
\hline & $\begin{array}{l}\text { Pregnant women } \\
N=105^{\mathrm{a}}\end{array}$ & $\begin{array}{l}\text { No pneumonia } \\
N=72\end{array}$ & $\begin{array}{l}\text { Pneumonia } \\
N=32\end{array}$ & $p$-value* & OR \\
\hline Age (years) & $34.1(28.8-37.1)$ & $34.3[28.5 ; 37.2]$ & $34.0[31.8 ; 36.4]$ & 0.778 & $1.01[0.94 ; 1.08]$ \\
\hline Ethnics & & & & 0.669 & \\
\hline Caucasian & $63(60 . \%)$ & $44(61.1 \%)$ & $18(56.2 \%)$ & & \\
\hline African & $2(1.9 \%)$ & $2(2.78 \%)$ & $0(0.00 \%)$ & & \\
\hline Arabic & $1(1 \%)$ & $1(1.39 \%)$ & $0(0.00 \%)$ & & \\
\hline LA & $30(28.6 \%)$ & 19 (26.4\%) & $11(34.4 \%)$ & & \\
\hline GA at diagnosis $(w)$ & $36.9(33.4-39.2)$ & $38.2[35.5 ; 39.8]$ & $34.3[28.2 ; 36.4]$ & $<0.001$ & $0.83[0.75 ; 0.92]$ \\
\hline Time of diagnosis & & & & 0.027 & \\
\hline Second trimester & $7(6.7 \%)$ & $2(2.78 \%)$ & $5(15.6 \%)$ & & Ref. \\
\hline Third trimester & $98(93.3 \%)$ & 70 (97.2\%) & $27(84.4 \%)$ & & $0.16[0.02 ; 0.85]$ \\
\hline Blood group & & & & 0.640 & \\
\hline 0 & $46(43.8 \%)$ & $32(44.4 \%)$ & $13(40.6 \%)$ & & Ref. \\
\hline A & $42(40.0 \%)$ & $29(40.3 \%)$ & $13(40.6 \%)$ & & $1.1[0.43 ; 2.78]$ \\
\hline B & $10(9.5 \%)$ & 5 (6.94\%) & $5(15.6 \%)$ & & $2.46[0.59 ; 10.3]$ \\
\hline$A B$ & $5(4.8 \%)$ & $4(5.56 \%)$ & $1(3.12 \%)$ & & $0.61[0.03 ; 4.67]$ \\
\hline Rh Positive & $91(86.7 \%)$ & $64(88.9 \%)$ & $27(84.4 \%)$ & 0.314 & $0.51[0.14 ; 1.96]$ \\
\hline Any comorbidity & 36 (34.3\%) & $20(27.8 \%)$ & $15(46.9 \%)$ & 0.093 & $2.27[0.95 ; 5.48]$ \\
\hline Symptomatology & $68(64.8 \%)$ & $35(48.6 \%)$ & $32(100 \%)$ & $<0.001$ & \\
\hline Pneumonia COVID-19 & $32(30.8 \%)$ & & & & \\
\hline Treatment & $46(43.8 \%)$ & $14(19.4 \%)$ & $32(100 \%)$ & $<0.001$ & \\
\hline Need of ICU admission & $5(4.8 \%)$ & $0(0.00 \%)$ & $5(15.6 \%)$ & 0.002 & \\
\hline Days at ICU & $10.0[6.5-18.5]$ & & $10.0[6.5-18.5]$ & & \\
\hline RT-PCR+ at delivery & $65(61.9 \%)$ & $45(62.5 \%)$ & $19(59.4 \%)$ & 0.933 & $0.88[0.37 ; 2.10]$ \\
\hline Caesarean section & $38(36.2 \%)$ & $18(25.0 \%)$ & $20(62.5 \%)$ & 0.004 & $4.88[2.02 ; 12.4]$ \\
\hline GA at delivery (w) & $39.0[37.4 ; 40.0]$ & $39.2[38.4 ; 40.1]$ & $38.4[34.1 ; 39.9]$ & 0.006 & $0.74[0.62 ; 0.89]$ \\
\hline Preterm birth & $21(20.6 \%)$ & $8(11.6 \%)$ & $13(40.6 \%)$ & 0.002 & $5.08[1.85 ; 14.9]$ \\
\hline
\end{tabular}

LA Latin American, GA Gestational Age, $w$ weeks, ICU Intensive Care Unit, RT-PCR Real Time- Polymerase Chain Reaction, $w$ weeks. ${ }^{\text {IIt }}$ was not possible to determine pneumonia or no pneumonia in one pregnant women. ${ }^{*} p$-value was calculated using Chi-squared or Fisher test for categorical variables and U-Mann Whitney for continuous variables

\section{Effects of SARS-CoV-2 during pregnancy in newborns} Overall, $53.3 \%$ of newborns were women and median weight at delivery was $3.050 \mathrm{~kg}$ (IQR: 2780-3455) with $5.6 \%$ of newborns were small for gestational age (Table 4). Median gestational age at delivery was 39.0 (IQR: 37.6-40.0) weeks with a range from 24 to 41 weeks. Apgar score at 1 and $5 \mathrm{~min}$ ranged from 1 to 9 with a median of 9.0 (IQR: 9.0-9.0) and 3 to 10 with a median of 10.0 (IQR: 10.0-10.0). Prematurity rate was $20.2 \%$. From all newborns, $16.8 \%$ required admission to a neonatal intensive care unit with a median duration of 3.0 (IQR: 1.0-8.0) days. None of the newborns presented any symptom of SARS-CoV-2 infection. The most common complications were due to prematurity. $66.4 \%$ of the newborns were breastfed.

An extreme premature newborn (24 week) died at 20 days of life due to prematurity-related complications.
Another full-term newborn died during the first $24 \mathrm{~h}$ from delivery, due to Sudden infant death Syndrome. Both newborns were born from women with severe pneumonia and admitted at the ICU.

Potential predictors for prematurity were diagnosed during the second trimester of pregnancy, having a positive RT-PCR at delivery, pneumonia during pregnancy and caesarean delivery in the univariable analysis. Having a positive RT-PCR at delivery and COVID-19 pneumonia during pregnancy were predictor factors for prematurity in the multivariable analysis (Fig. 2).

\section{Perinatal transmission of SARS-CoV-2 infection}

No vertical transmission was detected. Nasopharyngeal RT-PCR was possible to perform at $24-48 \mathrm{~h}$ of life in 101 newborns and repeated at 15 days old. None newborn tested positive at birth and one of the 61 (1.6\%) 
12

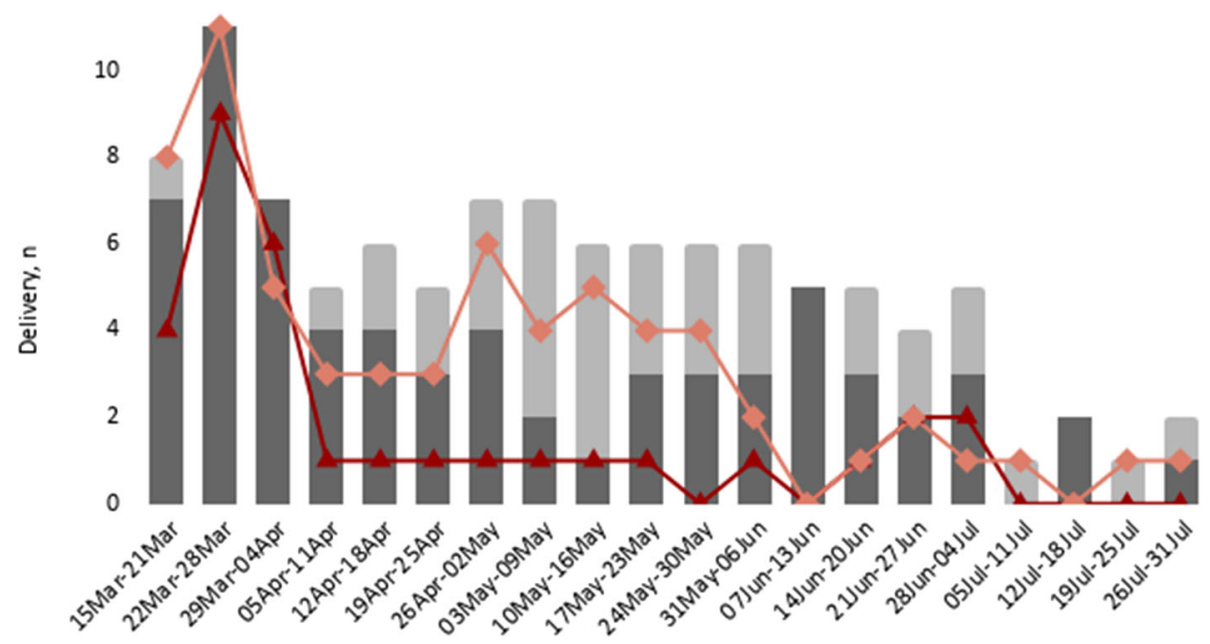

Fig. 1 Positive RT-PCR, pneumonia and symptomatology in pregnant women at delivery during the study period (15 March 2020-31 July 2020)

neonates tested at 15 days of life had a positive result with a previous negative result at birth. This case was a full-term newborn whose mother tested positive for SARS-CoV-2 RT-PCR during the $24 \mathrm{~h}$ previous to admission. Delivery was by caesarean section due to worsened maternal diseases, and after delivery, the mother was admitted at the ICU with COVID-19 pneumonia. The newborn was discharged after testing negative for SARS-CoV-2 RT-PCR at birth and lived with her asymptomatic grandmother, after the 15 days' diagnosis the neonate was tested positive for 2 SARS-CoV-2 RT-PCR for 2 weeks and under telephonic follow-up. That child was not breastfed, so this case was considered an intrafamily transmission.

\section{Discussion}

SARS-CoV-2 infection during pregnancy could cause COVID-19 pneumonia, that could condition an alteration during the pregnancy. In our series, pregnant women with COVID-19 pneumonia had a higher risk of caesarean sections ( $p=0.007$; OR $=4.20$, IC95\%: $1.47-11.99)$ and premature newborns ( $p=0.002$; OR $=6.97$, IC95\% 2.3422.75) than those not developing pneumonia.

COVID-19 pneumonia has been associated with gestational age at diagnosis, the older gestational age, the lower risk of pneumonia, for each week that increases gestational age, there is a $21 \%$ lower risk of pneumonia. It has also been associated with lymphopenia, having lymphopenia is 11 times more risk of pneumonia than not having it. The rate of severe pneumonia in pregnant women with SARS-CoV-2 infection in our study was $33 \%$, increasing the rate reported in a review from Juan et al. $^{7}(0-14 \%)$. The high rate of pneumonia diagnosed in this cohort could be explained because only patients with the moderate-severe disease were diagnosed and asymptomatic pregnant women delivering during the first month were not included at the beginning of the pandemic. Laboratory findings were consistent with values reported in non-pregnant adults with SARS-CoV2 infection including elevated inflammatory index such as C-reactive protein and fibrinogen.

We report a high rate of caesarean sections with a higher risk in pregnant women with pneumonia (4 times more risk). In our results, we find a $36.2 \%$ of caesarean section deliveries, close to $41.5 \%$ reported by Khoury et al. [32], which is increased compared with the latest upload of caesarean sections data in Spain (26.6\%) during 2015 (Instituto Nacional de Estadística, INE base, 2015). The rate of preterm births has been 20.6\%, increased compared to $14.6 \%$ reported by Khoury et al. [32] and definitively increased compared with the Spanish rate in 2015 (8.18\%) (Instituto Nacional de Estadística, INE base, 2015). This data supports Sentilhes et al. [10] and Li $\mathrm{N}$ et al. [33] reporting a higher rate of preterm delivery in infected women compared with noninfected women. In our study women developing pneumonia had 7 times more risk of preterm birth than women not developing pneumonia.

Elevated liver enzymes and D-dimer results have been found in women with pneumonia, as it was described before in other Spanish hospitals [11] but in our series, it has not been associated with pneumonia. Treatments used were safe for pregnant women and their newborns. All pregnant women diagnosed with SARS-CoV-2 received heparin for ten to fourteen days by protocol and there were no thromboembolic complications. 
Table 2 Summary of analytic findings by groups of pneumonia COVID-19

\begin{tabular}{|c|c|c|c|c|c|}
\hline & $\begin{array}{l}\text { Pregnant women } \\
N=105^{\mathrm{a}}\end{array}$ & $\begin{array}{l}\text { No pneumonia } \\
N=72\end{array}$ & $\begin{array}{l}\text { Pneumonia } \\
N=32\end{array}$ & $p$-value* & OR \\
\hline Glucose (mg/dL) & $86.0[78.0 ; 99.0]$ & $80.0[76.0 ; 88.0]$ & $94.0[84.8 ; 116]$ & 0.013 & $1.00[0.99 ; 1.01]$ \\
\hline Sodium (mmol/L) & $137[135 ; 138]$ & $137[136 ; 139]$ & $136[134 ; 137]$ & 0.014 & $0.80[0.64 ; 1.00]$ \\
\hline Potassium (mmol/L) & $4.00[3.77 ; 4.14]$ & $4.10[3.90 ; 4.20]$ & $3.85[3.60 ; 4.07]$ & 0.008 & $0.10[0.02 ; 0.62]$ \\
\hline Calcium (mg/dL) & $8.35[8.20 ; 8.47]$ & $8.40[8.20 ; 8.40]$ & $8.30[8.20 ; 8.50]$ & 1.000 & $1.01[0.66 ; 1.53]$ \\
\hline AST (U/L) & $54.0[21.5 ; 155]$ & $23.5[18.2 ; 151]$ & $78.0[34.0 ; 146]$ & 0.118 & $1.00[1.00 ; 1.01]$ \\
\hline ALT (U/L) & $19.0[13.0 ; 40.0]$ & $16.0[12.0 ; 25.0]$ & $24.0[16.0 ; 52.5]$ & 0.038 & $1.00[1.00 ; 1.01]$ \\
\hline GGT (U/L) & $13.5[8.00 ; 28.8]$ & $13.0[9.50 ; 24.2]$ & $18.0[7.75 ; 32.0]$ & 0.710 & $1.01[0.99 ; 1.03]$ \\
\hline Urea (mg/dL) & $16.0[12.8 ; 19.0]$ & $16.0[13.0 ; 18.0]$ & 16.0 [13.2;19.0] & 0.691 & $1.09[0.86 ; 1.38]$ \\
\hline Creatinine $(\mathrm{mg} / \mathrm{dL})$ & $0.59[0.49 ; 0.68]$ & $0.60[0.52 ; 0.66]$ & $0.56[0.48 ; 0.70]$ & 0.963 & $2.40[0.06 ; 92.8]$ \\
\hline LDH (U/L) & $210[177 ; 270]$ & $212[170 ; 266]$ & 209 [182;284] & 0.677 & $1.00[1.00 ; 1.00]$ \\
\hline CPK (units/L) & $47.0[31.0 ; 113]$ & $47.0[37.0 ; 113]$ & 49.0 [26.5;99.2] & 0.469 & $1.00[0.98 ; 1.01]$ \\
\hline CRP (mg/dL) & $4.60[1.75 ; 8.86]$ & $4.00[1.30 ; 9.20]$ & $5.00[2.15 ; 8.57]$ & 0.930 & $1.00[1.00 ; 1.01]$ \\
\hline Ferritin (mcg/L) & $47.0[30.0 ; 76.0]$ & $38.0[22.2 ; 48.5]$ & $73.0[42.5 ; 238]$ & 0.101 & $1.00[1.00 ; 1.01]$ \\
\hline D-dimer (ng/mL) & $637[438 ; 1325]$ & $864[616 ; 1844]$ & $493[404 ; 660]$ & 0.011 & $1.00[1.00 ; 1.00]$ \\
\hline Nt-proBNP (ng/L) & $269[163 ; 1128]$ & & $269[163 ; 1128]$ & & \\
\hline Fibrinogen $(\mathrm{mg} / \mathrm{dL})$ & $629[560 ; 694]$ & $610[567 ; 676]$ & $642[551 ; 712]$ & 0.518 & $1.00[1.00 ; 1.01]$ \\
\hline Troponin (ng/L) & $1.60[1.60 ; 2.40]$ & $1.60[1.60 ; 2.80]$ & $1.60[1.60 ; 2.15]$ & 0.718 & $1.01[0.88 ; 1.17]$ \\
\hline Procalcitonin (mcg/L) & $0.06[0.02 ; 0.10]$ & $0.03[0.02 ; 0.06]$ & $0.06[0.04 ; 0.10]$ & 0.072 & $1447[0.01 ; 172,837,716]$ \\
\hline Platelets (cells $/ \mathrm{mm}^{3}$ ) & $1300[900 ; 1800]$ & 1600 [1200;2100] & $950[725 ; 1200]$ & $<0.001$ & $1.00[1.00 ; 1.00]$ \\
\hline Haemoglobin $(g / d L)$ & $198,000[162,250 ; 240,250]$ & $204,000[164,500 ; 234,500]$ & $193,000[146,250 ; 261,000]$ & 0.909 & $1.00[1.00 ; 1.00]$ \\
\hline
\end{tabular}

AST Aspartate Aminotransferase, ALT Alanine Aminotransferase, GGT Gamma Glutamyltransferase, LDH Lactate Dehydrogenase, CPK Creatinine Phosphokinase, CRP C Reactive Protein, $n T$-proBNP N-terminal pro-B-type Natriuretic Peptide. ${ }^{a} / t$ was not possible to determine pneumonia or no pneumonia in one pregnant women. ${ }^{*} \mathrm{p}-$ overall was calculated using U-Mann Whitney for continuous variables

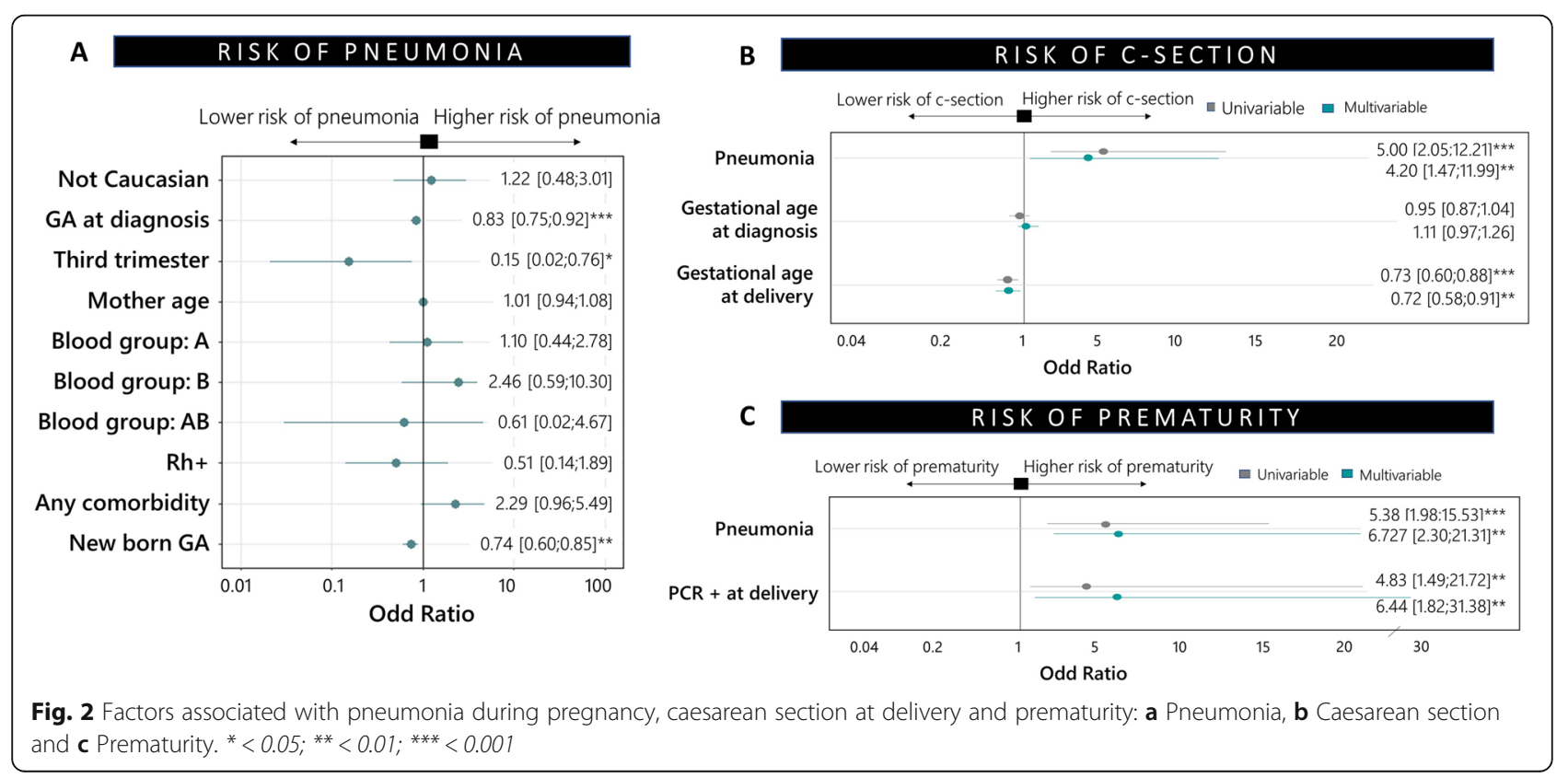


Table 3 Characteristics of women exposed to SARS-CoV-2 during gestation by c-section

\begin{tabular}{|c|c|c|c|c|c|}
\hline & $\begin{array}{l}\text { Pregnant women } \\
N=105\end{array}$ & $\begin{array}{l}\text { No C-section } \\
N=67\end{array}$ & $\begin{array}{l}\text { C-section } \\
N=32\end{array}$ & $p$-value* & OR \\
\hline Age (years) & $34.1[28.9 ; 37.0]$ & $34.9[30.9 ; 36.9]$ & $33.1[27.8 ; 36.9]$ & 0.233 & $0.95[0.89 ; 1.02]$ \\
\hline Ethnics & & & & 0.766 & \\
\hline Caucasian & $63(65.6 \%)$ & $41(68.3 \%)$ & $22(61.1 \%)$ & & Ref. \\
\hline African & $2(2.08 \%)$ & $1(1.67 \%)$ & $1(2.78 \%)$ & & \\
\hline Arabic & $1(1.04 \%)$ & $1(1.67 \%)$ & $0(0.00 \%)$ & & \\
\hline LA & $30(31.2 \%)$ & 17 (28.3\%) & $13(36.1 \%)$ & & \\
\hline GA at diagnosis (w) & $36.9[33.6 ; 39.1]$ & $37.6[34.2 ; 39.4]$ & $36.6[31.7 ; 39.0]$ & 0.325 & $0.95[0.87 ; 1.04]$ \\
\hline Time of diagnosis & & & & 0.096 & \\
\hline Second trimester & 7 (6.67\%) & $2(2.99 \%)$ & $5(13.2 \%)$ & & Ref. \\
\hline Third trimester & $98(93.3 \%)$ & $65(97.0 \%)$ & $33(86.8 \%)$ & & $0.21[0.03 ; 1.10]$ \\
\hline Blood group & & & & 0.488 & \\
\hline 0 & $46(44.7 \%)$ & $31(47.0 \%)$ & $15(40.5 \%)$ & & Ref. \\
\hline A & $42(40.8 \%)$ & $28(42.4 \%)$ & $14(37.8 \%)$ & & $1.03[0.42 ; 2.55]$ \\
\hline B & 10 (9.71\%) & $5(7.58 \%)$ & $5(13.5 \%)$ & & $2.04[0.48 ; 8.71]$ \\
\hline$A B$ & $5(4.85 \%)$ & $2(3.03 \%)$ & $3(8.11 \%)$ & & $2.96[0.41 ; 27.7]$ \\
\hline Rh Positive & 91 (88.3\%) & $59(89.4 \%)$ & $32(86.5 \%)$ & 0.752 & $0.76[0.22 ; 2.82]$ \\
\hline Any comorbidity & $36(34.3 \%)$ & $24(35.8 \%)$ & $12(31.6 \%)$ & 0.821 & $2.27[0.95 ; 5.48]$ \\
\hline Symptomatology & $68(64.8 \%)$ & 38 (56.7\%) & $30(78.9 \%)$ & 0.038 & $2.81[1.15 ; 7.48]$ \\
\hline Pneumonia COVID-19 & $32(30.8 \%)$ & $12(18.2 \%)$ & $20(52.6 \%)$ & $<0.001$ & $4.88[2.02 ; 12.4]$ \\
\hline Treatment & $59(56.2 \%)$ & $43(64.2 \%)$ & $16(42.1 \%)$ & 0.047 & $0.41[0.18 ; 0.93]$ \\
\hline Need of $\mathrm{UCl}$ admission & $5(4.76 \%)$ & $1(1.49 \%)$ & $4(10.5 \%)$ & 0.056 & $6.94[0.92 ; 195]$ \\
\hline Days at ICU & $10.0[10.0 ; 14.0]$ & $10.0[10.0 ; 10.0]$ & $12.0[8.25 ; 16.2]$ & 0.717 & $1.07[0.74 ; 1.54]$ \\
\hline RT-PCR+ at delivery & $65(61.9 \%)$ & $39(58.2 \%)$ & $26(68.4 \%)$ & 0.409 & $1.54[0.67 ; 3.68]$ \\
\hline GA at delivery $(w)$ & $39.0[37.6 ; 40.0]$ & $39.4[38.4 ; 40.1]$ & $38.1[34.6 ; 39.9]$ & 0.003 & $0.73[0.60 ; 0.88]$ \\
\hline Preterm birth & $21(20.6 \%)$ & $7(10.9 \%)$ & $14(36.8 \%)$ & 0.004 & $4.62[1.69 ; 13.8]$ \\
\hline
\end{tabular}

C-section Caesarean section, LA Latin American, GA Gestational Age, $w$ weeks, ICU Intensive Care Unit, RT-PCR Real Time- Polymerase Chain Reaction, $w$ weeks. ${ }^{*} p$ value was calculated using Chi-squared or Fisher test for categorical variables and U-Mann Whitney for continuous variables

Vertical transmission has not been objective and a horizontal transmission case was detected in this study. Even positivity on RT-PCR testing, the newborn did not present any symptomatology at the diagnosis nor during the follow-up. As Buonsenso et al. [19] have reported previously, we report a case of late onset infection in a 15 days old baby born from a woman infected during pregnancy. Maternal breastfeeding has been discarded as a source of transmission because the mother was admitted at the ICU with severe pneumonia, so it has been speculated an intra-family transmission. SARS-CoV-2 could be transmitted to newborns by close contact when not using appropriate hygiene measures [34]. Our study reinforces the national and international recommendations based on not modifying the type of delivery, not separating the mother from the newborn at birth and promoting breastfeeding as well as recommending to maximize hygiene by performing isolation of the contact in the environment.
Our results suggest that gestational age at diagnosis is associated with developing pneumonia, so it could be recommended to implement SARS-CoV-2 infection screening during pregnancy.

All treatment options received by infected women were safe for both women and newborns. Remdesivir has not been used during pregnancy in our cohort, both women receiving remdesivir was after delivery.

Maternal breastfeeding was indicated to infected women in our cohort following national recommendations and we have not detected breastfeeding transmission. The case of the newborn infected horizontally at home highlight the need to follow hygienically measures with newborns at home to avoid intrafamiliar infection. Additional research is required about clinical implications of SARS-CoV-2 infection during pregnancy.

There is a high research gap in SARS-CoV-2 infection during pregnancy. It would be interesting to elucidate if 
Table 4 Characteristics of newborns exposed to SARS-CoV-2 during gestation by prematurity

\begin{tabular}{|c|c|c|c|c|c|}
\hline & $\begin{array}{l}\text { Newborns }^{a} \\
N=107\end{array}$ & $\begin{array}{l}\text { No preterm } \\
N=72\end{array}$ & $\begin{array}{l}\text { Preterm } \\
N=32\end{array}$ & $p$-value* & OR \\
\hline Men & $47(46.7 \%)$ & $44(53.0 \%)$ & $12(57.1 \%)$ & 0.968 & $0.87[0.32 ; 2.31]$ \\
\hline Breastfeed & & & & 0.003 & \\
\hline Maternal & $71(66.4 \%)$ & $59(72.8 \%)$ & $9(42.9 \%)$ & & Ref. \\
\hline Artificial & $18(16.8 \%)$ & $9(11.1 \%)$ & $9(42.9 \%)$ & & $6.33[1.97 ; 21.2]$ \\
\hline Mixed & $16(15.0 \%)$ & $13(16.0 \%)$ & $3(14.3 \%)$ & & $1.54[0.29 ; 6.21]$ \\
\hline Apgar 1' & $9.00[9.00 ; 9.00]$ & $9.00[9.00 ; 9.00]$ & $9.00[8.00 ; 9.00]$ & 0.054 & $0.63[0.39 ; 1.02]$ \\
\hline Apgar 5' & $10.0[10.0 ; 10.0]$ & $10.0[10.0 ; 10.0]$ & $10.0[9.00 ; 10.0]$ & 0.009 & $0.52[0.28 ; 0.98]$ \\
\hline Small for GA & $6(5.61 \%)$ & $5(6.02 \%)$ & $1(4.76 \%)$ & 1.000 & $0.86[0.03 ; 6.05]$ \\
\hline Symptomatology & $81(75.7 \%)$ & 69 (83.1\%) & $9(42.9 \%)$ & $<0.001$ & $0.16[0.05 ; 0.44]$ \\
\hline Need of NICU Admission & $18(16.8 \%)$ & $6(7.23 \%)$ & $12(57.1 \%)$ & $<0.001$ & $17.6[5.36 ; 65.4]$ \\
\hline Days at NICU & $3.00[1.00 ; 8.00]$ & $1.00[0.50 ; 1.50]$ & $6.50[2.75 ; 14.8]$ & 0.015 & $1.61[0.91 ; 2.85]$ \\
\hline Oxygen need & $13(12.1 \%)$ & $4(4.82 \%)$ & $9(42.9 \%)$ & 0.203 & $3.44[0.54 ; 26.6]$ \\
\hline Days of oxygen & $6.00[1.00 ; 19.0]$ & $5.50[1.00 ; 10.8]$ & $9.00[1.00 ; 29.0]$ & 0.470 & $1.05[0.95 ; 1.16]$ \\
\hline Surfactant need & $6(5.61 \%)$ & $1(1.20 \%)$ & $5(23.8 \%)$ & 0.333 & $3.70[0.40 ; 118]$ \\
\hline RT-PCR at birth & & & & 0.344 & \\
\hline Negative & $101(94.4 \%)$ & 77 (92.8\%) & $21(100 \%)$ & & Ref. \\
\hline Not realized & $6(5.61 \%)$ & $6(7.23 \%)$ & $0(0.00 \%)$ & & \\
\hline RT-PCR at 15 days & & & & 0.700 & \\
\hline Negative & $60(56.1 \%)$ & $44(53.0 \%)$ & $13(61.9 \%)$ & 0.700 & Ref. \\
\hline Positive & $1(0.93 \%)$ & $1(1.20 \%)$ & $0(0.00 \%)$ & & \\
\hline
\end{tabular}

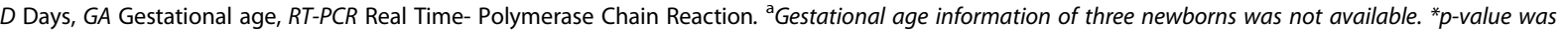
calculated using Chi-squared or Fisher test for categorical variables and U-Mann Whitney for continuous variables

there are potential long-term effects in women infected during pregnancy or possible consequences in newborns exposed to the virus during gestation. There is controversial data regarding the severity of SARS-CoV-2 infection during pregnancy; in our cohort, there is no mortality. Some studies have reported similar outcomes in infected pregnant women compared to non-pregnant adults with COVID-19 [35], but others have reported an increase in morbidity [36].

There are still unanswered questions, for example, what are the implications for women infected during the first trimester? Is there any consequence for newborns exposed to SARS-CoV-2 in a long term? These are important issues to clarify.

The most relevant limitation of our study is that at the beginning of the pandemic, the criteria for testing in Spain, and later by the neighbouring countries, only included patients with significant disease, which implicated the loss of asymptomatic infected women at delivery during the first month. All this, taking into account this is the largest multicentre study analysing both mother and newborn exposed to SARS-CoV-2 infection characteristics and outcomes and following newborns after birth.

\section{Conclusions}

As a conclusion, SARS-CoV-2 infection when symptomatic in pregnant women originated from admissions to the hospital during gestation and increased the time of admission of those with symptoms at delivery, women with pneumonia admitted to the ICU were at risk of maternal death. Even though the vertical transmission has not been reported in our cohort, the prognosis of newborns could be worsened by SARS-CoV-2 infection during pregnancy as we have seen COVID-19 pneumonia increases caesarean section deliveries and preterm births, increasing morbidity and mortality risks for women and newborns.

\section{Abbreviations}

SARS-CoV-2: Severe acute respiratory syndrome coronavirus 2; COVID19: Coronavirus disease 2019; ICU: Intensive care unit; RT-PCR: Real Time Polymerase Chain Reaction; SGA: Small for gestational age; IQR: Interquartile ranges, $\mathrm{OR}=$ Odds ratio

\section{Acknowledgements}

This study has been addressed on behalf of GESNEO COHORT WORKING GROUP: Teresa Hernández-Sampelayo Matos, Santiago Lizarraga Bonelli, Elena Rincón, Jesús Saavedra, Mar Santos, Begoña Santiago, Arantxa Berzosa, Ángela Manzanares, Laura Calle, Alba Pérez, María Ángeles Muñoz, Elena Vázquez, Laura Tarancón, María Jesús Fernández Aceñero, Lara Mesones, Andrea Martínez, Pilar Catalán, Roberto Fernández (Hospital 
General Universitario Gregorio Marañón); Amalia Rodelgo (Hospital Universitario Infanta Sofía); Inmaculada Lara, Álvaro Solaz (Hospital La Fe); Eva María Fernández (Hospital Materno Infantil de Badajoz); Mercedes Herranz, Amaya Pérez (Complejo Hospitalario de Navarra); Raquel López (Hospital Universitario de Albacete); Gloria Caro (Hospital Universitario Infanta Elena); Marta Sardá (Hospital Parc Tauli and Ana María Baña (Complejo Hospitalario de Santiago).

We would like to thank the Instituto de Salud Carlos III - Spanish Ministry of Science and Innovation for the financial support.

We thank HIV BioBank and microbiology Laboratory from Hospital General Universitario Gregorio Marañón for the sample processing, midwives and nurses from all participating hospitals for sample collection and patients and their families for their kindness.

We thank Charles Casillas Pérez for kindly reviewing the manuscript.

\section{Authors' contributions}

I.C., D.A., A.H. and M.N. have contributed with the design of the study; I.C., M.M., S.V., D.A., C.H., C.S., C.O., M.R., M.P., O.S., B.P., A.H., J.L., E.M. and M.N. have collected data; I.C., D.A. and S.D. have contributed with the statistical analysis; I. C and S. D have prepared tables and figures, I.C., S.V., D.A., S.D., M.N. interpreted the results; I.C. has writing the main manuscript text; M.M., S.V., D.A., C.H., C.S., C.O., M.R., M.P., O.S., B.P., J.L., E.M., S.D., J.L., M.S. and M.N. have reviewed the manuscript and M.N. has given the final approval of the manuscript.

\section{Funding}

This study was supported by the Instituto de Salud Carlos III - Spanish

Ministry of Science and Innovation (Grant n COV20/00808).

\section{Availability of data and materials}

The datasets used and/or analysed during the current study are available from the corresponding author on reasonable request.

\section{Declarations}

\section{Ethics approval and consent to participate}

The study was approved by the Ethics Committee from Hospital Gregorio Marañón and patients informed consent were obtained before inclusion. This study was conducted following WMA Declaration of Helsinki, a statement of ethical principles for medical research involving human subjects.

\section{Consent for publication}

Not applicable.

\section{Competing interests}

The authors declare that they have no competing interests.

\section{Author details \\ ${ }^{1}$ Paediatric Infectious Disease Unit, Instituto de Investigación Sanitaria Gregorio Marañón, Madrid, Spain. ${ }^{2}$ Facultad de Medicina Universidad Complutense de Madrid, Madrid, Spain. ${ }^{3}$ Department of Obstetrics and Gynecology, Hospital General Universitario Gregorio Marañón, Madrid, Spain. ${ }^{4}$ Department of Neonatology, Hospital General Universitario Gregorio Marañón, Madrid, Spain. ${ }^{5}$ Department of Paediatric Infectious Diseases, Hospital General Universitario Gregorio Marañón, Madrid, Spain. ${ }^{6}$ Department of Paediatric Gastroenterology Diseases, Hospital General Universitario Gregorio Marañón, Madrid, Spain. ${ }^{7}$ Department of Paediatrics, Hospital Universitario HM Montepríncipe, Madrid, Spain. ${ }^{8}$ Department of Paediatrics, Hospital General de Albace, Castilla La Mancha, Spain. ${ }^{9}$ Department of Obstetrics and Gynecology, Complejo Hospitalario de Navarra, Navarra, Spain. ${ }^{10}$ Department of Neonatology, Hospital Universitario Infanta Sofía, Madrid, Spain. ${ }^{11}$ Department of Paediatrics, Hospital Universitario Infanta Leonor, Madrid, Spain. ${ }^{12}$ Department of Paediatrics, Hospital San Pedro de Alcántara, Cáceres, Spain. ${ }^{13}$ Paediatric Infectious Diseases Unit, Fundación para la Investigación Biomédica del Hospital 12 de Octubre, Madrid, Spain.}

Received: 26 November 2020 Accepted: 22 March 2021 Published online: 26 April 2021

\section{References}

1. Velavan TP, Meyer CG. The COVID-19 epidemic. Tropical Med Int Health. 2020;25(3):278-80. https://doi.org/10.1111/tmi.13383.
2. Bulut C, Kato Y. Epidemiology of covid-19. Turk J Med Sci. 2020;50(SI-1):56370. https://doi.org/10.3906/sag-2004-172.

3. Schwartz DA. The effects of pregnancy on women with COVID-19: maternal and infant outcomes. doi:https://doi.org/10.1093/cid/ciaa559/5835848

4. Mei Y, Luo D, Wei S, Liao X, Pan Y, Yang X, et al. Obstetric management of COVID-19 in pregnant women. Front Microbiol. 2020;11. https://doi.org/1 0.3389/fmicb.2020.01186

5. Schwartz DA, Graham AL. Potential maternal and infant outcomes from coronavirus 2019-NCOV (SARS-CoV-2) infecting pregnant women: Lessons from SARS, MERS, and other human coronavirus infections. Viruses. 2020; 12(2). https://doi.org/10.3390/v12020194.

6. Hantoushzadeh S, Shamshirsaz AA, Aleyasin A, et al. Maternal death due to COVID-19. Am J Obstet Gynecol. 2020;223(1):109.e1-109.e16. https://doi. org/10.1016/j.ajog.2020.04.030.

7. Juan J, Gil MM, Rong Z, Zhang Y, Yang H, Poon LC. Effects of coronavirus disease 2019 ( $<$ scp $>$ COVID $</$ scp $>-19$ ) on maternal, perinatal and neonatal outcomes: a systematic review. Ultrasound Obstet Gynecol. 2020: uog.22088. https://doi.org/10.1002/uog.22088.

8. Rothan HA, Byrareddy SN. The epidemiology and pathogenesis of coronavirus disease (COVID-19) outbreak. J Autoimmun. 2020;109:102433. https://doi.org/10.1016/j.jaut.2020.102433.

9. Garg S, Kim L, Whitaker M, et al. MMWR - hospitalization rates and characteristics of patients hospitalized with laboratory-confirmed coronavirus disease 2019 - COVID-NET, 14 States, March 1-30, 2020.; 2019. https://www.cdc.gov/coronavirus/2019-ncov/

10. Sentilhes $L$, de Marcillac F, Jouffrieau C, et al. Coronavirus disease 2019 in pregnancy was associated with maternal morbidity and preterm birth. Am J Obstet Gynecol. 2020. https://doi.org/10.1016/j.ajog.2020.06.022.

11. San-Juan R, Barbero P, Fernández-Ruiz M, López-Medrano F, Lizasoáin M, Hernández-Jiménez $P$, et al. Incidence and clinical profiles of COVID19 pneumonia in pregnant women: a single-Centre cohort study from Spain. EClinicalMedicine. 2020;23:100407. https://doi.org/10.1016/j. eclinm.2020.100407

12. Egloff C, Vauloup-Fellous C, Picone O, Mandelbrot L, Roques P. Evidence and possible mechanisms of rare maternal-fetal transmission of SARS-CoV-2. J Clin Virol. 2020;128:104447. https://doi.org/10.1016/j.jcv.2020.104447.

13. Yu N, Li W, Kang Q, Xiong Z, Wang S, Lin X, et al. Clinical features and obstetric and neonatal outcomes of pregnant patients with COVID-19 in Wuhan, China: a retrospective, single-Centre, descriptive study. Lancet Infect Dis. 2020;20(5):559-64. https://doi.org/10.1016/S1473-3099(20)30176-6.

14. Yang Z, Liu Y. Vertical transmission of severe acute respiratory syndrome coronavirus 2: a systematic review. Am J Perinatol. 2020;37(10):1055-60. https://doi.org/10.1055/s-0040-1712161.

15. Ashraf MA, Keshavarz $P$, Hosseinpour $P$, et al. Systematic review coronavirus disease 2019 (COVID-19): A systematic review of pregnancy and the possibility of vertical transmission, vol. 21; 2020.

16. Alzamora MC, Paredes T, Caceres D, Webb CM, Valdez LM, la Rosa M. Severe COVID-19 during pregnancy and possible vertical transmission. Am J Perinatol. 2020;37(8):861-5. https://doi.org/10.1055/s-0040-1710050.

17. Vivanti AJ, Vauloup-Fellous C, Prevot $\mathrm{S}$, et al. Transplacental transmission of SARS-CoV-2 infection. Nat Commun. 2020;11(1). https://doi.org/10.1038/s414 67-020-17436-6

18. Zeng L, Xia S, Yuan W, Yan K, Xiao F, Shao J, et al. Neonatal early-onset infection with SARS-CoV-2 in 33 neonates born to mothers with COVID-19 in Wuhan, China. JAMA Pediatr. 2020;(7):722-5. https://doi.org/10.1001/jama pediatrics.2020.0878

19. Buonsenso D, Costa S, Sanquinetti M, Cattani P, Posteraro B, Marchetti S, et al. Neonatal late onset infection with severe acute respiratory syndrome coronavirus 2. Am J Perinatol. 2020;37(8):869-72. https://doi.org/10.1055/s0040-1710541.

20. Demirjian A, Singh C, Tebruegge M, Herbert R, Draz N, Mirfenderesky M, et al. Probable vertical transmission of SARS-CoV-2 infection. Pediatr Infect Dis J. 2020;39(9):e257-60. https://doi.org/10.1097/INF.0000000000002821.

21. Wang S, Guo L, Chen L et al. A Case Report of Neonatal 2019 Coronavirus Disease in China. Clin Infect Dis. 2020;71(15):853-7. https://doi.org/10.1093/ cid/ciaa225.

22. Sisman J, Jaleel MA, Moreno W. Intrauterine Transmission of SARS-COV-2 Infection in Preterm Infant. Pediatr Infect Dis J. 2020;39(9):e265-e267. https:// doi.org/10.1097/INF.0000000000002815.

23. Penfield CA, Brubaker SG, Limaye MA, Lighter J, Ratner AJ, Thomas KM, et al. Detection of SARS-COV-2 in Placental and Fetal Membrane Samples. Am J 
Obstetr Gynecol MFM. 2020;(3):100133. https://doi.org/10.1016/j.ajogmf.202 0.100133.

24. Verma S, Carter EB, Mysorekar IU. SARS-CoV2 and pregnancy: an invisible enemy? Am J Reprod Immunol. 2020;17(5):e13308. https://doi.org/10.1111/a ji.13308.

25. Algarroba GN, Rekawek P, Vahanian SA, Khullar P, Palaia T, Peltier MR, et al. Visualization of severe acute respiratory syndrome coronavirus 2 invading the human placenta using electron microscopy. Am J Obstet Gynecol. 2020;223(2): 275-8. https://doi.org/10.1016/j.ajog.2020.05.023

26. Qiu $H, W u J$, Hong $L$ et al. Clinical and epidemiological features of 36 children with coronavirus disease 2019 (COVID-19) in Zhejiang, China: an observational cohort study. Lancet Infect Dis. 2020;20(6):689-96. https://doi. org/10.1016/S1473-3099(20)30198-5. Epub 2020 Mar 25.

27. Salvatore CM, Han J-Y, Acker KP, et al. Neonatal management and outcomes during the COVID-19 pandemic: an observation cohort study. Lancet Child Adolesc Health. 2020. https://doi.org/10.1016/\$2352-4642(20)3 0235-2.

28. Parazzini F, Bortolus R, Mauri PA, Favilli A, Gerli S, Ferrazzi E. Delivery in pregnant women infected with SARS-CoV-2: a fast review. Int J Gynecol Obstet. 2020;9. https://doi.org/10.1002/ijgo.13166.

29. Dhochak N, Singhal T, Kabra SK, Lodha R. Pathophysiology of COVID-19: why children fare better than adults? Indian J Pediatr. 2020;87(7):537-46. https://doi.org/10.1007/s12098-020-03322-y.

30. Spanish Society of Neonatology. Recommendations for management of newborns for SARS-CoV-2 infection. 2020. Version 6.0. Date 13/04/2020.

31. Harris PA, Taylor R, Minor BL, Elliott V, Fernandez M, O'Neal L, et al. The REDCap consortium: building an international community of software platform partners. J Biomed Inform. 2019;95:103208. https://doi.org/10.1016/ j.jbi.2019.103208

32. Khoury R, Bernstein PS, Debolt C, et al. Characteristics and outcomes of 241 births to women with severe acute respiratory syndrome coronavirus 2 (SARS-CoV-2) infection at five New York City medical centers. Obstet Gynecol. 2020. https://doi.org/10.1097/AOG.0000000000004025.

33. Li N, Han L, Peng M et al. Maternal and Neonatal Outcomes of Pregnant Women With Coronavirus Disease 2019 (COVID-19) Pneumonia: A CaseControl Study. Clin Infect Dis. 2020;71(16):2035-41. https://doi.org/10.1093/ $\mathrm{cid} / \mathrm{ciaa352}$.

34. Fernández-Carrasco FJ, María Vázquez-Lara J, González-Mey U, GómezSalgado J, Parrón-Carreño T, Rodríguez-Díaz L. Infección por coronavirus covid-19 y lactancia materna: una revisión exploratoria revisión panorámica. 2020;94 www.mscbs.es/resp.

35. Molteni E, Astley CM, Ma W, et al. SARS-CoV-2 (COVID-19) infection in pregnant women: characterization of symptoms and syndromes predictive of disease and severity through real-time, remote participatory epidemiology. doi:https://doi.org/10.1101/2020.08.17.20161760

36. Phoswa WN, Khaliq OP. Is pregnancy a risk factor of COVID-19? Eur J Obstet Gynecol Reprod Biol. 2020:605-9. https://doi.org/10.1016/j.ejogrb.2020.06. 058.

\section{Publisher's Note}

Springer Nature remains neutral with regard to jurisdictional claims in published maps and institutional affiliations.

Ready to submit your research? Choose BMC and benefit from:

- fast, convenient online submission

- thorough peer review by experienced researchers in your field

- rapid publication on acceptance

- support for research data, including large and complex data types

- gold Open Access which fosters wider collaboration and increased citations

- maximum visibility for your research: over $100 \mathrm{M}$ website views per year

At $\mathrm{BMC}$, research is always in progress.

Learn more biomedcentral.com/submissions 